\title{
ON THE REDUCIBILITY OF CERTAIN QUADRINOMIALS
}

\author{
JONAS JANKAUSKAS \\ Vilnius University, Lithuania
}

\begin{abstract}
In 2007 West Coast Number Theory conference Walsh asked to determine all irreducible polynomials of the form $P(x)=x^{i}+$ $x^{j}+x^{k}+4$ with integer exponents $i>j>k>0$, such that for some positive integer $l$ the polynomial $P\left(x^{l}\right)$ is reducible in $\mathbb{Z}[x]$. In this paper we prove that such polynomials are quadrinomials $x^{4 m}+x^{3 m}+x^{2 m}+4$, where $m$ is an odd positive integer. In addition, Walsh asked for the examples of reducible quadrinomials $x^{i}+x^{j}+x^{k}+n, n>4$ with no linear or quadratic factors. We compute the examples of reducible polynomials of the form above with non-trivial factors and negative coefficient $n$.
\end{abstract}

\section{INTRODUCTION}

Let $P(x)$ be a polynomial with integer coefficients. The polynomial $P(x)$ is called primitive, if one cannot write $P(x)=P_{1}\left(x^{l}\right)$ for some polynomial $P_{1} \in \mathbb{Z}[x]$ and integer $l>1$. The reciprocal polynomial $x^{\operatorname{deg} P} P(1 / x)$ of the polynomial $P(x)$ is denoted by $P^{*}(x)$. Through the paper, reducibility shall always mean reducibility in $\mathbb{Z}[x]$.

The following question was posed by Walsh ([9]) in 2007 at West Coast Number Theory conference (Problem $007: 14$ ). Let $i>j>k$ be positive integers. Does there exist an irreducible polynomial $P(x)=x^{i}+x^{j}+x^{k}+4$ of degree $\operatorname{deg} P>17$, such that for some integer $l>1$, the polynomial $P\left(x^{l}\right)$ factors in $\mathbb{Z}[x]$ ?

In addition, Walsh asked for the examples of reducible primitive quadrinomials of the form $x^{i}+x^{j}+x^{k}+n$ with integer constant coefficient $n>4$, which have no linear or quadratic factors. He gave one such example $x^{7}+x^{5}+x^{3}+8=\left(x^{3}-x^{2}-x+2\right)\left(x^{4}+x^{3}+3 x^{2}+2 x+4\right)$.

2010 Mathematics Subject Classification. $12 \mathrm{E} 05$.

Key words and phrases. Reducibility, quadrinomials. 
The choice of the constant coefficient 4 in the polynomial $x^{i}+x^{j}+x^{k}+4$ is not accidental. A similar example is the binomial $x^{2}+4$ which factors after the change of variable $x$ to $x^{2}$. The polynomials $x^{4 m}+4 b^{4}$ are the exceptional case in the theorem of Capelli $([12])$ on the reducibility of binomials. In the trinomial case, all reducible polynomials of the form $x^{i} \pm x^{j} \pm 4$ were completely determined by Jonassen $([6])$. By the theorem given in his paper [6], there are no irreducible trinomials $P(x)=x^{i} \pm x^{j} \pm 4$, such that for some positive integer $l$, the polynomial $P\left(x^{l}\right)$ is reducible. In contrast, there exist quadrinomials $P(x)=x^{i}+x^{j}+x^{k}+4$ which have this property. In Section 2 , we shall give a complete description of such quadrinomials.

The questions on the reducibility of trinomials and quadrinomials have received a lot of interest. The reducible trinomials and quadrinomials with all non-zero coefficients equal to 1 or -1 were investigated by Selmer ([13]) and Ljunggren ([7]). The missing cases in Ljunggren's work were settled by Mills ([8]). Many important results and generalizations were established by Schinzel in the long series of papers starting with [10,11]. In 1972 Fried and Schinzel ([5]) proved a deep result on the reducibility of quadrinomials. Theorems 2 and 3 in [5] state that for the fixed integers $a, b, c, d$, any reducible quadrinomial $P(x)=a x^{i}+b x^{j}+c x^{k}+d$ either factors into the product of certain polynomials of standard shape, or such polynomial has the form $P(x)=P_{1}\left(x^{l}\right), l \in \mathbb{Z}, l>0$, were $P_{1} \in \mathbb{Z}[x]$ is primitive reducible quadrinomial of degree less or equal to the effectively computable constant $C(a, b, c, d)$. Unfortunately, this constant is too large for almost any practical applications: in our case, $C(1,1,1,4)>2^{8045222}$. In the present paper, we shall use Ljunggren's method ([7]) to determine all such exceptional quadrinomials $x^{i}+x^{j}+x^{k}+4$ which appear in the question of Walsh. See $[1,4]$ for a good exposition on the Ljunggren's method. More recent results on reducibility of trinomials can be found in [3]. For efficient factoring algorithms, we refer to $[2]$.

\section{MAIN RESULTS}

The following theorem gives an answer to the first question of Walsh. We note that in this paper we do not use the same terminology as in [9].

THEOREM 2.1. The only primitive irreducible polynomial $P \in \mathbb{Z}[x]$ of the form $P(x)=x^{i}+x^{j}+x^{k}+4, i>j>k>0$, such that the polynomial $P\left(x^{l}\right)$ for some positive integer $l$ factors in $\mathbb{Z}[x]$, is the polynomial $P(x)=x^{4}+x^{3}+x^{2}+4$. More precisely, for $l=2$,

$P\left(x^{2}\right)=x^{8}+x^{6}+x^{4}+4=\left(x^{4}-x^{3}+x^{2}-2 x+2\right)\left(x^{4}+x^{3}+x^{2}+2 x+2\right)$.

Indeed, if the polynomial $P(x)=x^{i}+x^{j}+x^{k}+4$ has the property asked in the Problem $007: 14$, then $P(x)=P_{1}\left(x^{m}\right)$ for some primitive polynomial $P_{1}(x)$ and some positive integer $m$. By Theorem 2.1, $P_{1}(x)=x^{4}+x^{3}+x^{2}+4$. 
In Lemma 2.2 bellow, we prove that $P\left(x^{l}\right)$ is reducible only for even values $l=2 g$; in this case the polynomial $P\left(x^{l}\right)$ splits into two irreducible factors $x^{4 g}-x^{3 g}+x^{2 g}-2 x^{g}+2$ and $x^{4 g}+x^{3 g}+x^{2 g}+2 x^{g}+2$.

LEMMA 2.2. Let $P(x)=x^{d}+a_{d-1} x^{d-1}+\cdots+a_{1} x+a_{0}$ be a monic integer polynomial, such that $\left|a_{0}\right|=p^{2}>\left|a_{1}\right|+\left|a_{2}\right|+\cdots+\left|a_{d-1}\right|+1$, where $p$ is a prime. Let $l>1$ be a positive integer, such that $P\left(x^{l}\right)$ is reducible in $\mathbb{Z}[x]$ but $P\left(x^{m}\right)$ is irreducible for any positive integer $m<l$ which divides $l$. Then $l$ is even and $P\left(x^{l}\right)= \pm Q(x) Q(-x), Q \in \mathbb{Z}[x]$. Moreover, for any integer $r \geqslant 1$, both factors $Q\left( \pm x^{r}\right) \in \mathbb{Z}[x]$ are irreducible.

In Lemma 2.3 we shall determine all the possible forms the product polynomial $P P^{*}$ can take. This will be used in the proof of Theorem 2.1.

Lemma 2.3. Let $P$ be a quadrinomial $P(x)=x^{i}+x^{j}+x^{k}+4$ with the integer exponents $i>j>k>0$. Then the polynomial PP* takes one of the following forms:

1) $4 x^{2 i}+x^{2 i-k}+x^{2 i-j}+x^{i+j-k}+4 x^{i+j}+4 x^{i+k}+19 x^{i}+4 x^{i-k}+4 x^{i-j}+$ $x^{i-j+k}+x^{j}+x^{k}+4$, if $\quad i \neq 2 j, \quad i \neq 2 k, \quad j \neq 2 k, \quad i+k \neq 2 j, \quad i \neq j+k$;

2) $4 x^{2 i}+x^{2 i-k}+5 x^{2 i-j}+x^{i+j-k}+4 x^{i+k}+19 x^{i}+4 x^{i-k}+x^{i-j+k}+5 x^{j}+$ $x^{k}+4$,

if $\quad i=2 j, \quad i \neq 2 k, \quad j \neq 2 k, \quad i+k \neq 2 j, \quad i \neq j+k$;

3) $4 x^{2 i}+5 x^{2 i-k}+x^{2 i-j}+x^{i+j-k}+4 x^{i+j}+19 x^{i}+4 x^{i-j}+x^{i-j+k}+x^{j}+$ $5 x^{k}+4$,

if $\quad i \neq 2 j, \quad i=2 k, \quad j \neq 2 k, \quad i+k \neq 2 j, \quad i \neq j+k$;

4) $4 x^{2 i}+x^{2 i-k}+x^{2 i-j}+5 x^{i+j-k}+4 x^{i+j}+19 x^{i}+4 x^{i-j}+5 x^{i-j+k}+x^{j}+$ $x^{k}+4$,

if $\quad i \neq 2 j, \quad i \neq 2 k, \quad j=2 k, \quad i+k \neq 2 j, \quad i \neq j+k$;

5) $4 x^{2 i}+x^{2 i-k}+2 x^{2 i-j}+4 x^{i+j}+4 x^{i+k}+19 x^{i}+4 x^{i-k}+4 x^{i-j}+2 x^{j}+x^{k}+4$, if $\quad i \neq 2 j, \quad i \neq 2 k, \quad j \neq 2 k, \quad i+k=2 j, \quad i \neq j+k$;

6) $4 x^{2 i}+5 x^{2 i-k}+5 x^{2 i-j}+x^{i+j-k}+19 x^{i}+x^{i-j+k}+5 x^{j}+5 x^{k}+4$, if $\quad i \neq 2 j, \quad i \neq 2 k, \quad j \neq 2 k, \quad i+k \neq 2 j, \quad i=j+k$;

7) $4 x^{2 i}+x^{2 i-k}+5 x^{2 i-j}+5 x^{i+j-k}+19 x^{i}+5 x^{i-j+k}+5 x^{j}+x^{k}+4$, if $\quad i=2 j, \quad i \neq 2 k, \quad j=2 k, \quad i+k \neq 2 j, \quad i \neq j+k$;

8) $4 x^{2 i}+5 x^{2 i-k}+2 x^{2 i-j}+4 x^{i+j}+19 x^{i}+4 x^{i-j}+2 x^{j}+5 x^{k}+4$, if $\quad i \neq 2 j, \quad i=2 k, \quad j \neq 2 k, \quad i+k=2 j, \quad i \neq j+k$;

9) $4 x^{2 i}+5 x^{2 i-k}+6 x^{2 i-j}+19 x^{i}+6 x^{j}+5 x^{k}+4$, if $\quad i \neq 2 j, \quad i \neq 2 k, \quad j=2 k, \quad i+k=2 j, \quad i=j+k$. 


\section{Computations}

In order to answer the second part of the problem $007: 14$, we have used the computer to search for the examples of reducible polynomials of the form $P(x)=x^{i}+x^{j}+x^{k}+n$. Since the polynomial $P(x)$ has no roots of modulus less or equal to 1 if $n \geqslant 4$, the polynomial $P(x)$ is irreducible provided the coefficient $n$ is equal to the prime integer $p \geqslant 5$. With MAPLE computer algebra package we factored all primitive quadrinomials $P(x)$ with composite constant coefficient $n$ and exponents $i, j, k$ in the range $5<n \leqslant 120, i-j \leqslant 20$, $j-k \leqslant 20, k \leqslant 20$. In addition, we factored all primitive quadrinomials $P(x)$ of this form satisfying inequalities $120<n \leqslant 1000, i-j \leqslant 15, j-k \leqslant 15$, $k \leqslant 15$. We also searched for the irreducible polynomials $P(x)$, such that $P\left(x^{l}\right)$ is reducible for some integer $l$ in the range $5<n \leqslant 120,(i-j) l \leqslant 12$, $(j-k) l \leqslant 12, k l \leqslant 12$. In all the cases reducible polynomials $P(x)$ had a factor of the form $Q\left(x^{l}\right)$, where $Q(x)$ was a linear polynomial or a quadratic polynomial. The example $x^{7}+x^{5}+x^{3}+8$ of Walsh was the only notable exception. However, it does not seem easy to prove this.

All found examples of reducible quadrinomials $P(x)$ had two or three irreducible factors. During the preparation of this paper, A. Schinzel sent a short remark that any irreducible polynomial dividing the quadrinomial $P(x)$ has constant coefficient greater than 1 , hence the number of irreducible factors cannot exceed $\Omega(n)$, the total number of prime factors of $n$. The sharpness of this estimate may be shown by the example

$$
x^{12}+x^{8}+x^{4}+52=\left(x^{2}-2 x+2\right)\left(x^{2}+2 x+2\right)\left(x^{8}-3 x^{4}+13\right) .
$$

Since $\Omega(n) \leqslant \log n / \log 2$, the number $\log n / \log 2$ is the best known bound for the total number of prime factors of the quadrinomials $P(x)$ in question.

Finally, we note that there exist the examples of reducible quadrinomials $P(x)$ with no linear or quadratic factors and negative coefficient $n<-5$, namely, the polynomials

$$
\begin{aligned}
x^{6}+x^{4}+x^{2}-16 & =\left(x^{3}-3 x^{2}+5 x-4\right)\left(x^{3}+3 x^{2}+5 x+4\right), \\
x^{12}+x^{8}+x^{4}-16 & =\left(x^{3}-x^{2}-x+2\right)\left(x^{3}+x^{2}-x-2\right)\left(x^{6}+3 x^{4}+5 x^{2}+4\right), \\
x^{7}+x^{3}+x^{2}-98 & =\left(x^{3}-x^{2}+2 x-7\right)\left(x^{4}+x^{3}-x^{2}+4 x+14\right),
\end{aligned}
$$

and

$$
\begin{aligned}
x^{17}+x^{14}+x^{8}-16= & \left(x^{5}+x^{3}-x^{2}-2\right) \\
& \left(x^{12}-x^{10}+2 x^{9}+x^{8}-x^{7}+x^{6}+2 x^{4}+4 x^{3}-4 x^{2}+8\right) .
\end{aligned}
$$

\section{Proofs}

Proof of Lemma 2.2. Let $P\left(x^{l}\right)=Q(x) R(x)$ for some integer $l>0$ and polynomials $Q, R \in \mathbb{Z}[x]$. The inequality $\left|a_{0}\right|>\left|a_{1}\right|+\left|a_{2}\right|+\cdots+\left|a_{d_{1}}\right|+1$ implies that $P, Q$ and $R$ have no roots of modulus $|z| \leqslant 1$. Thus the constant 
terms of $Q$ and $R$ are equal to $\pm p$ and they are irreducible in $\mathbb{Z}[x]$. Otherwise one of the polynomials $Q$ or $R$ would be divisible by the monic non-constant polynomial $S \in \mathbb{Z}[x]$ with the constant term $S(0)= \pm 1$. This is impossible, since such a polynomial $S$ has at least one root of modulus less or equal to 1 . The same argument also implies the irreducibility of polynomials $Q\left(x^{r}\right)$ and $R\left(x^{r}\right)$ which divide $P\left(x^{r l}\right)$.

Now, assume that the exponent $l$ has the property that for any positive integer $m<l$ which divides $l$, the polynomial $P\left(x^{m}\right)$ is irreducible. If $m=1$, this means that $P(x)$ is irreducible. Let $\alpha$ be the root of the irreducible factor $Q(x)$. Since $P\left(x^{l}\right)=Q(x) R(x)$, the power of this root $\beta=\alpha^{l}$ is the root of the irreducible polynomial $P$. Let $K=\mathbb{Q}(\beta), L=\mathbb{Q}(\alpha), K \subset L$. Let $g$ be the degree $[L: K]$. The absolute norm of an algebraic integer $\beta$ over $\mathbb{Q}$ $N_{L / \mathbb{Q}}(\beta)=N_{L / \mathbb{Q}}\left(\alpha^{l}\right)=N_{L / \mathbb{Q}}(\alpha)^{l}= \pm p^{l}$. In the other hand, by the relative norm property, $N_{L / \mathbb{Q}}(\beta)=N_{K / \mathbb{Q}}(\beta)^{[L: K]}= \pm p^{2 g}$. Thus $l=2 g$.

Since $l$ is even, the number $-\alpha$ is the root of $P\left(x^{l}\right)$. The irreducible polynomial $Q(-x)$ divides $P\left(x^{l}\right)$. Then $R(x)= \pm Q(-x)$. Indeed, otherwise $Q(-x)=-Q(x)$ or $Q(-x)=Q(x)$. The first case is impossible: the identity $Q(-x)=-Q(x)$ with $x=0$ implies $Q(0)=P(0)=0$, contradicting the inequality $\left|a_{0}\right|>1$. The second identity $Q(-x)=Q(x)$ implies $Q(x)=T\left(x^{2}\right)$ for the polynomial $T \in \mathbb{Z}[x]$, thus $R(x)=P\left(x^{l}\right) / Q(x)=P\left(x^{2 g}\right) / T\left(x^{2}\right)=$ $S\left(x^{2}\right), S \in \mathbb{Z}[x]$. This leads to the expression $P\left(x^{2 g}\right)=T\left(x^{2}\right) S\left(x^{2}\right)$, hence $P\left(x^{g}\right)=T(x) S(x)$. This implies that $P\left(x^{g}\right)$ is reducible for the integer $g$ wich is a proper divisor of $l$, contradicting the condition of Lemma 2.2.

Proof of Lemma 2.3. Assume that integers $i, j, k$ satisfy the inequality $i>j>k>0$. The reciprocal of the polynomial $P(x)=x^{i}+x^{j}+x^{k}+4$ is $P^{*}(x)=4 x^{i}+x^{i-k}+x^{i-j}+1$. The product $F=P P^{*}$ takes the form

$$
\begin{aligned}
F(x)= & 4 x^{2 i}+x^{2 i-k}+x^{2 i-j}+4 x^{i+j}+x^{i+j-k}+4 x^{i+k} \\
& +19 x^{i}+4 x^{i-k}+x^{i-j+k}+4 x^{i-j}+x^{j}+x^{k}+4 .
\end{aligned}
$$

Let

$$
\begin{gathered}
v_{1}=2 i, \quad v_{2}=2 i-k, \quad v_{3}=2 i-j, \quad v_{4}=i+j, \quad v_{5}=i+j-k \\
v_{6}=i+k, \quad v_{7}=i, \quad v_{8}=i-k, \quad v_{9}=i-j+k, \quad v_{10}=i-j \\
v_{11}=j, \quad v_{12}=k, \quad v_{13}=0
\end{gathered}
$$

The multi-set $V=\left\{v_{r}, r=0, \ldots, 13\right\}$ contains all possible exponents which appear in the polynomial $F$. If none of them are equal, $F$ takes the form in Case 1). We shall classify all other cases where some exponents $v_{r}$ and $v_{s}, r \neq s$ are equal, and the terms of $F$ with equal exponents add together. Set

$$
\begin{gathered}
e_{1}=\{i=2 j\}, \quad e_{2}=\{i=2 k\}, \quad e_{3}=\{j=2 k\} \\
e_{4}=\{i+k=2 j\}, \quad e_{5}=\{i=j+k\}
\end{gathered}
$$


The elements of the set $E=\left\{e_{r}, r=1, \ldots, 5\right\}$ denote the linear relations among the integers $i, j, k$. Note that $v_{1}$ and $v_{13}$ are the largest and smallest integers in $V$. Since $i>j>k>0$, all the exponents $v_{1}, v_{2}, v_{3}, v_{4}, v_{5}, v_{6}$ are strictly greater than the exponent of the middle term $v_{7}=i$, exponents $v_{8}, v_{9}, v_{10}, v_{11}, v_{12}, v_{13}$ are strictly less than $v_{7}=i . F$ is self-reciprocal, since $F=P P^{*}=F^{*}$. Hence $v_{s}=2 i-v_{13-s+1}, s=8, \ldots, 13$. Thus it suffices to check all possible cases when some of the integers $v_{2}, v_{3}, v_{4}, v_{5}, v_{6}$ are equal. Observe that

$$
v_{2}>v_{3}, \quad v_{2}>v_{5}, \quad v_{4}>v_{5}, \quad v_{4}>v_{6} .
$$

Hence, all possible pairs of equal exponents $v_{s}, s=2, \ldots, 6$ are: $v_{2}=v_{4}$ (this is equivalent to the linear relation $\left.e_{5}\right), v_{2}=v_{6}$ (equivalent to $e_{2}$ ), $v_{3}=v_{4}$ $\left(e_{1}\right), v_{3}=v_{5}\left(e_{4}\right), v_{3}=v_{6}\left(e_{5}\right), v_{5}=v_{6}\left(e_{3}\right)$. The remaining pairs of equal exponents $v_{s}, s=8, \ldots, 13$ are determined uniquely by the symmetry $v_{s}=2 i-v_{13-s+1}$.

The forms of the polynomial $F$ where the integer exponents $i, j, k$ satisfy precisely one linear relation in the set $E$ are listed in Cases 2)-6). In order to check Cases 2)-6), use the expression in Case 1) and add terms with equal powers $x^{v_{r}}$ and $x^{v_{s}}$ together if $v_{r}=v_{s}$. The next step is to determine all possible forms of $F$ where the integers $i, j, k$ satisfy two linear relations $\left\{e_{s}, e_{t}\right\} \subset E$. Observe that no one pair of the linear relations $\left\{e_{1}, e_{2}\right\},\left\{e_{1}, e_{4}\right\},\left\{e_{1}, e_{5}\right\},\left\{e_{2}, e_{3}\right\},\left\{e_{2}, e_{5}\right\}$ is possible if $i>j>k>0$. The possible pairs are $\left\{e_{1}, e_{3}\right\},\left\{e_{2}, e_{4}\right\},\left\{e_{3}, e_{4}\right\},\left\{e_{3}, e_{5}\right\},\left\{e_{4}, e_{5}\right\}$. Consider the pair $\left\{e_{1}, e_{3}\right\}$ as a system of two linear equations in three integer variables $i, j, k$. All positive integer solutions of this system are vectors $(i, j, k)=(4 u, 2 u, u), u \in \mathbb{Z}, u>0$. In this case, the form of the polynomial $F$ with equal exponents $v_{3}=v_{4}, v_{5}=v_{6}, v_{8}=v_{9}, v_{10}=v_{11}$ is described in Case 7) of Lemma 2.3. Similarly, the integer solutions to the equations $\left\{e_{2}, e_{4}\right\}$ are $(i, j, k)=(4 u, 3 u, 2 u), u \in \mathbb{Z}, u>0$ and $F$ takes the form given in Case $8)$. Observe that all the pairs of equations from the system $\left\{e_{3}, e_{4}, e_{5}\right\}$ are equivalent and have the solution $(i, j, k)=(3 u, 2 u, u), u \in \mathbb{Z}, u>0$. Hence, any two of the three relations $\left\{e_{3}, e_{4}, e_{5}\right\}$ imply the third one. This situation is depicted in Case 9). It remains to show that there are no other cases where three or more linear relations $e_{s} \in E$ hold. Indeed, in such case three different pairs of linear relations, other than all 3 possible pairs from the set $\left\{e_{3}, e_{4}, e_{5}\right\}$ must be satisfied. There would be at least one of pairs $\left\{e_{1}, e_{3}\right\},\left\{e_{2}, e_{4}\right\}$ and one pair from the set $\left\{e_{3}, e_{4}, e_{5}\right\}$. This is impossible, since all the intersections of the sets of integer triples $(i, j, k)$ which satisfy such linear relations

$$
\begin{gathered}
\{(4 u, 2 u, u), u \in \mathbb{Z}, u>0\}, \quad\{(4 u, 3 u, 2 u), u \in \mathbb{Z}, u>0\}, \\
\{(3 u, 2 u, u), u \in \mathbb{Z}, u>0\}
\end{gathered}
$$

are empty. 
Sketch of The PRoof of Theorem 2.1 Before proceed to prove Theorem 2.1, we give the sketch of the proof. First, we show that any primitive quadrinomial $P(x)=x^{i}+x^{j}+x^{k}+4$ in question whose exponents $(i, j, k)$ satisfy a certain linear relation is precisely the quadrinomial $x^{4}+x^{3}+x^{2}+4$. Secondly, we consider the polynomial $G(x)=Q(-x) Q^{*}(x)$, where $Q$ is the polynomial from the factorization $P\left(x^{l}\right)= \pm Q(x) Q(-x)$. This factorization is a consequence of Lemma 2.2. We determine the form of the polynomial $G$ using reduction modulo 2 and the identity $\|P\|=\|G\|$ for the Euclidean norms of $P$ and $G$. Recall that the Euclidean norm $\|f\|$ of the polynomial $f(x)=\sum_{j=1}^{\operatorname{deg} f} a_{j} x^{j} \in \mathbb{Z}[x]$ is defined as $\|f\|=\left(\sum_{j=1}^{\operatorname{deg} f} a_{j}^{2}\right)^{1 / 2}$. Following [7], we refer to the equality $\|P\|=\|G\|$ as the identity of Ljunggren. Thirdly, we use the expression $G G^{*}(x)=P P^{*}\left(x^{l}\right)$ and compare $P P^{*}\left(x^{l}\right)$ from Lemma 2.3 to $G G^{*}(x)$. We establish that the case $x^{4}+x^{3}+x^{2}+4$ is the only one possible.

Proof of Theorem 2.1. First suppose that the exponents $i, j, k$ of $P(x)=x^{i}+x^{j}+x^{k}+4$ satisfy linear relations $i=2 k$ and $i+k=2 j$. Then $(i, j, k)=(4 u, 3 u, 2 u)$ for some positive integer $u$ so $P(x)=x^{4 u}+x^{3 u}+x^{2 u}+4$, which is primitive for $u=1$. A simple computation shows that the polynomial $P_{1}(x)=x^{4}+x^{3}+x^{2}+4$ is irreducible, and $P_{1}\left(x^{2}\right)=\left(x^{4}-x^{3}+x^{2}-2 x+\right.$ $2)\left(x^{4}+x^{3}+x^{2}+2 x+2\right)$. For even integers $l=2 g>0$ the polynomial $P_{1}\left(x^{l}\right)$ splits in $\mathbb{Z}[x]$ into $P_{1}\left(x^{l}\right)=\left(x^{4 g}-x^{3 g}+x^{2 g}-2 x^{g}+2\right)\left(x^{4 g}+x^{3 g}+x^{2 g}+2 x^{g}+2\right)$. By Lemma 2.2, both factors are irreducible. By Lemma 2.2, $P_{1}\left(x^{l}\right)$ is irreducible for odd exponents $l$. Below we shall show that this case is the only possible. Note that the linear relations $i=2 k, i+k=2 j$ appear in the Case 8) of Lemma 2.3. Hence we have to prove that every quadrinomial $P(x)$ in the question of Walsh satisfies $P\left(x^{l}\right) P^{*}\left(x^{l}\right)=F\left(x^{l}\right)$, where $F$ is a polynomial in Case 8) of Lemma (2.3).

Let $P(x)=x^{i}+x^{j}+x^{k}+4$ be an irreducible polynomial and $l>0$ be an integer such that $P\left(x^{l}\right)$ splits in $\mathbb{Z}[x]$, while $P\left(x^{m}\right)$ is irreducible for any integer $m, 1 \leqslant m<l$ dividing the exponent $l$. By Lemma $2.2, l=2 g, g \in \mathbb{Z}$, and $P\left(x^{l}\right)= \pm Q(x) Q(-x)$. Without loss of generality, we may assume that $Q(x)$ is monic. Then $P\left(x^{l}\right)=(-1)^{i g} Q(x) Q(-x)$. The polynomials $Q(x)$ and $Q(-x)$ have equal constant terms \pm 2 , hence $P(x)=Q(x) Q(-x)$. This implies that the degree $i g$ is even. Also, $Q(0)=2$. Otherwise $Q(x)$ has a positive real root which is impossible, since $P(x)>0$ if $x>0$. Consider the reduction of $P\left(x^{2 g}\right)$ modulo 2 :

$$
P\left(x^{g}\right)^{2} \equiv P\left(x^{2 g}\right)=Q(x) Q(-x) \equiv Q(x)^{2} \quad(\bmod 2) .
$$

Hence $Q(x) \equiv P\left(x^{g}\right) \equiv x^{i g}+x^{j g}+x^{k g}(\bmod 2)$. Let $G$ be the product $G(x)=Q(-x) Q^{*}(x)$ of degree $2 i g$. Note that the polynomial $G$ satisfies the identity $x^{2 i g} G(1 / x)=(-1)^{i g} G(-x)$. Since $i g$ is even, $G^{*}(x)=G(-x)$. Hence the integer coefficients of $G(x)=\sum_{s=0}^{2 i g} b_{s} x^{s}$ which are symmetric with the 
respect of middle term are equal in modulus, more precisely,

$$
b_{s}=(-1)^{s} b_{2 i g-s}, 0 \leqslant s \leqslant 2 i g .
$$

Reduce $G(x)$ modulo 2:

$$
\begin{aligned}
G(x) & =Q^{*}(x) Q(-x) \equiv Q^{*}(x) Q(x) \equiv\left(x^{(i-k) g}+x^{(i-j) g}+1\right)\left(x^{i g}+x^{j g}+x^{k g}\right) \\
(4.2) & \equiv x^{(2 i-k) g}+x^{(2 i-j) g}+x^{(i+j-k) g}+x^{i g}+x^{(i-j+k) g}+x^{j g}+x^{k g}(\bmod 2) .
\end{aligned}
$$

Note that in $(4.2)$ the commuting of the operation $*$ and reduction $(\bmod 2)$ is essentially used, which make sense since $f^{*}(x)(\bmod 2)=(f(x)(\bmod 2))^{*}$ if and only if the leading coefficient of $f \in \mathbb{Z}[x]$ is odd.

Since $Q$ is monic, $Q(0)=2$, the leading and constant coefficients of $G$ are equal to 2 . Since $i>j>k>0$, the exponents in $G$ modulo 2 satisfy the inequalities

$$
(2 i-k) g>(2 i-j) g \geqslant(i+j-k) g>i g>(i-j+k) g \geqslant j g>k g,
$$

provided $i+k \geqslant 2 j$ or

$$
(2 i-k) g>(i+j-k) g \geqslant(2 i-j) g>i g>j g \geqslant(i-j+k) g>k g,
$$

provided $i+k \leqslant 2 j$. Hence the polynomial $G(x)$ in (4.2) has 7 odd coefficients if $i+k \neq 2 j$. If $i+k=2 j, G(x)$ modulo 2 takes the form

$$
G(x) \equiv x^{(2 i-k) g}+x^{i g}+x^{k g} \quad(\bmod 2),
$$

with 3 odd coefficients. Observe that $P\left(x^{l}\right) P^{*}\left(x^{l}\right)=G(x) G^{*}(x)$. The equality holds since $\left(Q^{*}\right)^{*}=Q$ which is true since $Q(0) \neq 0$. By the identity of Ljunggren, $\|G\|^{2}=\|P\|^{2}=1^{2}+1^{2}+1^{2}+4^{2}=19$. The leading and constant coefficients of $G$ are equal to 2 , thus the sum of squares of coefficients $b_{s}, 1 \leqslant$ $s \leqslant 2 i g-1$ is equal to 11 . In addition, there must be precisely 3 or 7 odd coefficients by (4.2) and (4.3). All such possible sums of squares are

(4.4) $11=3^{2}+1^{2}+1^{2}=2^{2}+2^{2}+1^{2}+1^{2}+1^{2}=2^{2}+1^{2}+1^{2}+1^{2}+1^{2}+1^{2}+1^{2}+1^{2}$

The absolute values of the coefficients of $G$ are symmetric with respect to the middle term $b_{i g} \equiv 1(\bmod 2)$. Thus an integer which appears in the sum of squares above the odd number of times must be the absolute value of the middle coefficient $b_{i g}$. Hence the summands in the third sum in (4.4) cannot be the squares of coefficients of the polynomial $G$. This implies that $G$ has 3 odd coefficients and the exponents $i, j, k$ satisfy the relation $i+k=2 j$. Using the identities (4.1), (4.3), and (4.4) we deduce that $G$ takes one of the forms:

$$
\begin{gathered}
G(x)=2 x^{2 i g}+\varepsilon x^{(2 i-k) g}+3 \delta x^{i g}+(-1)^{k g} \varepsilon x^{k g}+2, \\
G(x)=2 x^{2 i g}+\varepsilon_{1} x^{(2 i-k) g}+2 \varepsilon_{2} x^{t+i g}+\delta x^{i g} \\
+(-1)^{t} 2 \varepsilon_{2} x^{i g-t}+(-1)^{k g} \varepsilon_{1} x^{k g}+2,
\end{gathered}
$$

the coefficients $\varepsilon, \varepsilon_{1}, \varepsilon_{2}, \delta$ are all equal to -1 or 1 and $t$ is an integer $0<t<i g$. Also, note that the terms $x^{t+i g}, x^{i g-t}$ do not coincide with any other term 
of the polynomial $G$ in (4.6) by (4.4). We shall determine the coefficients $\varepsilon, \varepsilon_{1}, \varepsilon_{2}, \delta$. Observe that $G(1)=Q(1) Q(-1)=P(1)=7$. In (4.5), this is possible if and only if $\delta=1$ and $\varepsilon+(-1)^{k g} \varepsilon=0$, hence the exponent $k g$ is odd in (4.5). Moreover, we may assume that $\varepsilon=1$. Otherwise, replace $x$ by $-x$. Thus $G$ in (4.5) takes the form

(i) $2 x^{2 i g}+x^{(2 i-k) g}+3 x^{i g}-x^{k g}+2,2 \nmid k g$.

Consider $G$ in (4.6). There are three cases where $G(x)$ takes the value $G(1)=$ 7 :

(a) $2 \mid k g, 2 \nmid t, \varepsilon_{1}=1, \delta=1$. Assume $\varepsilon_{2}=1$, otherwise change $x$ to $-x$;

(b) $2 \mid t, 2 \nmid k g, \varepsilon_{2}=1, \delta=-1$. Assume $\varepsilon_{1}=1$, otherwise change $x$ to $-x$

(c) $2|\mathrm{~kg}, 2| t, \varepsilon_{1}=-1, \varepsilon_{2}=1, \delta=1$.

The polynomial $G$ in Cases (a),(b),(c) takes the forms (ii), (iii), (iv) below, respectively.

(ii) $2 x^{2 i g}+x^{(2 i-k) g}+2 x^{t+i g}+x^{i g}-2 x^{i g-t}+x^{k g}+2$,

(iii) $2 x^{2 i g}+x^{(2 i-k) g}+2 x^{t+i g}-x^{i g}+2 x^{i g-t}-x^{k g}+2$,

(iv) $2 x^{2 i g}-x^{(2 i-k) g}+2 x^{t+i g}+x^{i g}+2 x^{i g-t}-x^{k g}+2$.

In each case (i), (ii), (iii), (iv) we check if $G(x) G^{*}(x)=P\left(x^{2 g}\right) P^{*}\left(x^{2 g}\right)=$ $F\left(x^{2 g}\right)$, where $F(x)$ is one of the polynomials in Lemma 2.3. Since $i+k=2 j$, it suffices to check Cases 5), 8), 9) in Lemma 2.3. First, assume that $G$ takes the form (i). Then

$$
G(x) G^{*}(x)=4 x^{4 i g}+12 x^{3 i g}-x^{(4 i-2 k) g}+19 x^{2 i g}-x^{2 k g}+12 x^{i g}+4
$$

has 7 non-zero coefficients, hence it must coincide with $F\left(x^{2 g}\right)$ in Case 9) of Lemma 2.3. This is impossible, since the coefficients of $F$ are different from the coefficients of $G G^{*}$. Next, assume that $G$ takes the form (iii). Compute the product $G G^{*}$ modulo 4 :

$$
G(x) G^{*}(x) \equiv-x^{(4 i-2 k) g}-x^{2 i g}-x^{2 k g} \quad(\bmod 4) .
$$

None of the polynomials $F$ in Lemma 2.3 satisfy $F\left(x^{2 g}\right) \equiv G(x) G^{*}(x)$ (mod 4). Thus the form (iii) is impossible.

Assume that $G$ takes the form (iv). The integer $2 i g$ is largest exponent in $G$. Let $v$ be the second largest exponent in $G$. Clearly, $v=(2 i-k) g$ or $v=t+i g$. Observe that $2 i g+v>s+r$ if at least one inequality $r \leqslant 2 i g, s \leqslant v$ is strict. Hence the second largest exponent in $G G^{*}$ is $2 i g+v$. Thus the first two terms of $G G^{*}$ are $x^{4 i g}-4 x^{(4 i-k) g}$ if $(2 i-k) g>t+i g$ or $x^{4 i g}+8 x^{3 i g+t}$ if $(2 i-k) g<t+i g$. Such terms do not occur in any polynomial in Lemma 2.3. Hence we reject the form (iv). 
This implies that $G$ takes the form (ii). The product $G G^{*}$ is

$$
\begin{aligned}
G(x) G^{*}(x)= & 4 x^{4 i g}+4 x^{(4 i-k) g}-4 x^{2 t+2 i g}+x^{(4 i-2 k) g}+4 x^{3 i g}+2 x^{(3 i-k) g} \\
& +4 x^{(2 i+k) g}+19 x^{2 i g}+4 x^{(2 i-k) g}+2 x^{(i+k) g}+4 x^{i g}+x^{2 k g} \\
& -4 x^{2 i g-2 t}+4 x^{k g}+4 .
\end{aligned}
$$

Thus $G G^{*}$ coincides with the polynomial $F\left(x^{2 g}\right)$ in Case 5), 8) or 9) of Lemma 2.3 , since $i+k=2 j$ in (ii). Since $i>k>0$, the integer $4 i-k$ is strictly greater than $3 i, 4 i-2 k, 3 i-k, 2 i+k$. If $(4 i-k) g>2 i g+2 t$, then the second leading term of $G G^{*}$ is $4 x^{(4 i-k) g}$. This leads directly to the Case 8) of the Lemma 2.3. Indeed, only polynomials $F(x)$ in Case 5) or Case 8) have terms with coefficients equal to 4 which are not leading nor constant terms. The term with the second highest exponent $4 x^{(4 i-k) g}$ in $G G^{*}$ must coincide with the term $4 x^{2(i+j) g}$ or $4 x^{2(i+k) g}$ in $F\left(x^{2 g}\right)$ in Case 5). Since $j>k$, the exponent $2(i+j) g$ is greater than $2(i+k) g$. Thus $(4 i-k) g=2(i+j) g$, so $4 i-k=2 i+2 j$. Together with the identity $i+k=2 j$ in Case 5) the linear relation $4 i-k=2 i+2 j$ implies $i=2 k$, which implies Case 8) of Lemma 2.3.

Hence we may assume that $(4 i-k) g \leqslant 2 i g+2 t$. If the inequality is strict, then the second leading term of $G G^{*}$ is $-4 x^{2 i g+2 t}$. However, the polynomials in Lemma 2.3 have no negative terms. Hence $(4 i-k) g=2 i g+2 t$. Thus

$$
\begin{aligned}
G G^{*}= & 4 x^{4 i g}+x^{(4 i-2 k) g}+4 x^{3 i g}+2 x^{(3 i-k) g}+4 x^{(2 i+k) g} \\
& +19 x^{2 i g}+4 x^{(2 i-k) g}+2 x^{(i+k) g}+4 x^{i g}+x^{2 k g}+4 .
\end{aligned}
$$

Let $F(x)$ be the polynomial in Case 5) of Lemma 2.3. Replace $x$ by $x^{2 g}$ and use the identity $2 j=i+k$. The resulting polynomial is

$$
\begin{aligned}
F\left(x^{2 g}\right)= & 4 x^{4 i g}+x^{(4 i-2 k) g}+2 x^{(4 i-2 j) g}+4 x^{(2 i+2 j) g}+4 x^{(2 i+2 k) g} \\
& +19 x^{2 i g}+4 x^{(2 i-2 k) g}+4 x^{(2 i-2 j) g}+2 x^{2 j g}+x^{2 k g}+4 \\
= & 4 x^{4 i g}+x^{(4 i-2 k) g}+2 x^{(3 i-k) g}+4 x^{(3 i+k) g}+4 x^{(2 i+2 k) g} \\
& +19 x^{2 i g}+4 x^{(2 i-2 k) g}+4 x^{(i-k) g}+2 x^{(i+k) g}+x^{2 k g}+4 .
\end{aligned}
$$

The difference $F\left(x^{2 g}\right)-G G^{*}=4 x^{(3 i+k) g}+4 x^{(2 i+2 k) g}+4 x^{(2 i-2 k) g}+4 x^{(i-k) g}-$ $4 x^{3 i g}-4 x^{(2 i+k) g}-4 x^{(2 i-k) g}-4 x^{i g} \neq 0$, since the exponent $(3 i+k) g$ is the larger than other exponents in $F\left(x^{2 g}\right)-G G^{*}$. Thus $G G^{*}$ does not coincide with a polynomial given in Case 5) of Lemma 2.3.

Let $F(x)$ be the polynomial in Case 9) of Lemma 2.3. The equations $j=2 k, 2 j=i+k, i=j+k$ imply $(i, j, k)=(3 u, 2 u, u), u \in \mathbb{Z}, u>0$. Hence

$$
\begin{aligned}
G G^{*}= & 4 x^{12 u g}+x^{10 u g}+4 x^{9 u g}+2 x^{8 u g}+4 x^{7 g}+19 x^{6 u g}+4 x^{5 u g} \\
& +2 x^{4 u g}+4 x^{3 u g}+x^{2 u g}+4 .
\end{aligned}
$$


Also, $F\left(x^{2 g}\right)=4 x^{12 u g}+5 x^{10 u g}+6 x^{8 u g}+19 x^{6 u g}+6 x^{4 u g}+5 x^{2 u g}+4$ and $F\left(x^{2 g}\right) \neq G G^{*}$, so Case 9) is impossible. Hence we conclude that Case 8) is the only one possible. This completes the proof.

\section{ACKNOWLEDGEMENTS.}

We thank A. Schinzel for the remarks concerning the paper [5] and his note on the total number of irreducible factors of quadrinomials $x^{i}+x^{j}+x^{k}+n$. We are grateful to the referees for useful comments and corrections.

\section{REFERENCES}

[1] M. Filaseta, On the factorization of polynomials with small Euclidean norm, In: Number theory in progress (Zakopane-Koscielisko, 1997), de Gruyter, Berlin, 1 (1999), 143-163.

[2] M. Filaseta, A. Granville and A. Schinzel, Irreducibility and greatest common divisor algorithms for sparse polynomials, In: Number Theory and Polynomials (ed. James McKee and Chris Smyth), LMS Lecture Note Series 352, Cambridge Univ. Press, 2008, 155-176.

[3] M. Filaseta, F. Luca, P. Stănică and R. G. Underwood, Two Diophantine approaches to the irreducibility of certain trinomials, Acta Arith. 128 (2007), 149-156.

[4] M. Filaseta and I. Solan, An extension of a theorem of Ljunggren, Math. Scand. 84 (1999), 5-10.

[5] M. Fried and A. Schinzel, Reducibility of quadrinomials, Acta Arith. 21 (1972), 153171.

[6] A. T. Jonassen, On the irreducibility of the trinomials $x^{m} \pm x^{n} \pm 4$, Math. Scand. 21 (1967), 177-189.

[7] W. Ljunggren, On the reducibility of certain trinomials and quadrinomials, Math. Scand. 8 (1960), 65-70.

[8] W. H. Mills, The factorization of certain quadrinomials, Math. Scand. 57 (1985), $44-50$.

[9] G. Myerson, Western Number Theory Problems, 17-19 Dec 2007, 6. Available online at http://www.math.colostate.edu/ achter/wntc/problems/problems2007.pdf

[10] A. Schinzel, On the reducibility of polynomials and in particular of trinomials, Acta Arith. 11 (1965), 1-34.

[11] A. Schinzel, Reducibility of lacunary polynomials. I, Acta Arith. 16 (1969/1970), $123-159$.

[12] A. Schinzel, Polynomials with special regard to reducibility, Encyclopedia of Mathematics and its applications 77, Cambridge Univ. Press, 2000.

[13] E. S. Selmer, On the irreducibility of certain trinomials, Math. Scand. 4 (1956), 287-302.

\section{J. Jankauskas}

Department of Mathematics and Informatics

Vilnius University

Naugarduko 24, Vilnius LT-03225

Lithuania

E-mail: jonas.jankauskas@gmail.com

Received: 10.6.2009.

Revised: 19.6 .2009 . 